\title{
Optical and Magnetic Microstructures in YIG Ferrite Fabricated by Femtosecond Laser
}

\author{
Atsushi Ishikawa ${ }^{1,2}$, Tomohiro Amemiya ${ }^{3}$, Yuya Shoji ${ }^{4}$, Pham Nam Hai ${ }^{5}$, Masaaki Tanaka ${ }^{5}$, \\ Tetsuya Mizumoto ${ }^{4}$, Shigehisa $\mathrm{Arai}^{3,4}$, and Takuo Tanaka ${ }^{2,6}$ \\ ${ }^{1}$ Department of Electrical and Electronic Engineering, Okayama University, Japan \\ ${ }^{2}$ Metamaterials Laboratory, RIKEN, Japan \\ ${ }^{3}$ Quantum Nanoelectronics Research Center, Tokyo Institute of Technology, Japan \\ ${ }^{4}$ Department of Electrical and Electronic Engineering, Tokyo Institute of Technology, Japan \\ ${ }^{5}$ Department of Electrical Engineering and Information Systems, The University of Tokyo, Japan \\ ${ }^{6}$ Research Institute for Electronic Science, Hokkaido University, Japan \\ E-mail:a-ishikawa@okayama-u.ac.jp
}

\begin{abstract}
Optical and magnetic microstructures are directly fabricated inside a film of cerium-substituted yttrium iron garnet (Ce:YIG) by using a direct femtosecond laser writing. The laser irradiation induces local changes in both optical and magnetic properties of Ce:YIG. A quantitative phase imaging technique proves that the refractive index is increased by $0.015 \pm 0.001$, which is about $0.7 \%$ of Ce:YIG refrative index 2.2 at $632.8 \mathrm{~nm}$. The magneto-optical polar Kerr effect measurement shows that the magnetization property is changed from hard to soft, decreasing the coercivity. Our proposed technique paves the way toward the development of novel three-dimensional magneto-optical devices for optical communication and photonic applications.
\end{abstract}

DOI: $10.2961 / \mathrm{jlmn} .2015 .01 .0010$

Keywords: Femtosecond laser fabrication, Multi-photon absorption, Three-dimensional structures, Magneto-optical materials, Integrated optics.

\section{Introduction}

Femtosecond laser processing has recently been applied to a wide variety of fields, such as laser ablation, lithographic fabrication, and photo-reduction reaction [1-4]. Since this fabrication technique is based on nonlinear multi-photon absorption, it is possible to highly localize only at the focal voxel [5]. Successive chemical or physical reactions can be also confined in a small volume, allowing for both thee-dimensional (3D) and sub-diffraction $(\sim 100 \mathrm{~nm})$ resolutions in the fabrication [6,7]. By utilizing such unique abilities of the direct laser writing, there have been a number of reports on the formation of $3 \mathrm{D}$ micro/nanostructures in transparent materials [8-12] and the development of novel optical functional devices [13-16]. In those studies, although 3D photonic devices were nicely demonstrated based on the refractive index change induced by highintense laser pulses, glasses and polymers were mainly used as photo-reactive media and other optical materials are not well studied. To explore versatile applications of the direct laser writing, we here demonstrate femtosecond laser microstructuring of ferrimagnetic ceramic materials. Specifically, cerium-substituted yttrium iron garnet $\left(\mathrm{Ce}_{\mathrm{x}} \mathrm{Y}_{3}\right.$ ${ }_{x} \mathrm{Fe}_{5} \mathrm{O}_{12}$ : $\left.\mathrm{Ce}: \mathrm{YIG}\right)$ is used as a photo-reactive medium, and we show that laser irradiation modifies both the optical and magnetic properties of the irradiated Ce:YIG areas [17].

Ce:YIG is one of the most typical ferrimagnetic ceramic materials with a large Faraday rotation and high transparency at infrared wavelengths [18,19]. Because of its excellent magneto-optical performance [20], Ce:YIG has been widely used for nonlinear optical devices, such as Faraday rotators and non-reciprocal waveguide devices to break the time-reversal symmetry of light propagation in photonic integrated circuits (PICs) [21-24]. Although Ce:YIG has many potential applications in PICs, there is one major issue in the process of their fabrication; Ce:YIG is not inherently compatible with PICs consisting of semiconductor optical components. There, an adhesive agent or plasma-assisted direct bonding [25,26] are currently employed for YIG joining, but neither of these processes is sufficient for mechanical, chemical, and thermal durability. On the key issue of how one can incorporate optical and magnetic components into one device, our proposed technique is very appealing because optical and magnetic microstructures can be directly fabricated at one time without any additional process. Furthermore, even complex optical and magnetic stereostructures can be produced, thus being a potential approach to develop novel 3D magneto-optical devices for optical communication and photonic applications [27].

\section{Femtosecond laser fabrication in Ce:YIG}

Figure 1(a) shows a Ce:YIG wafer used for the experiment. A 1.2- $\mu$ m-thick single-crystalline Ce:YIG layer was epitaxially grown on a $<111>$-oriented nonmagnetic garnet substrate $\left((\mathrm{GdCa})_{3}(\mathrm{GaMgZr})_{5} \mathrm{O}_{12}\right.$ or SGGG) using magnetoron sputtering with a substrate temperature of $690{ }^{\circ} \mathrm{C}$ and an argon atmospheric pressure of $0.8 \mathrm{~Pa}$. The sample had a mirror-like surface with a yellowish green color and a relative refractive index of 2.2. Fig. 1(b) depicts the X-ray diffraction spectrum of the Ce:YIG/SGGG wafer. Very sharp Bragg reflections from a Ce:YIG $<888>$ plane are clearly resolved with $\mathrm{K} \alpha 1$ and $\mathrm{K} \alpha 2$ lines, demonstrating high crystallographic quality of the grown Ce:YIG layer. The linear 

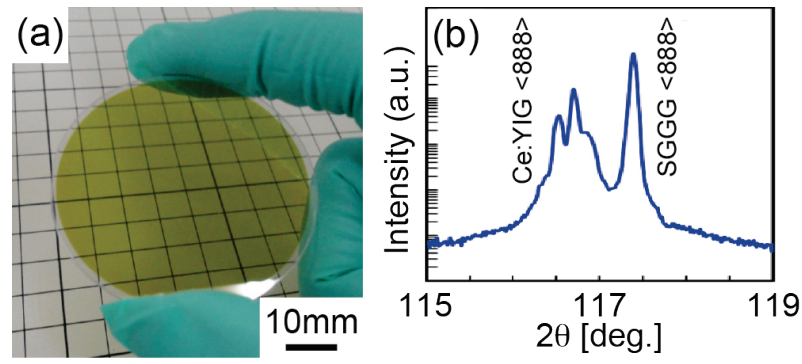

(c)

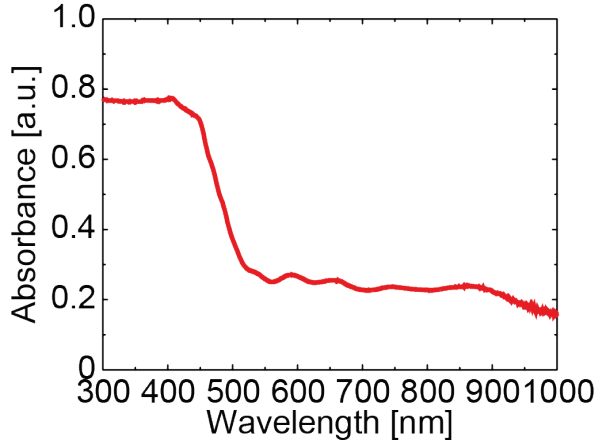

Fig. 1 (a) 1.2- $\mu \mathrm{m}$-thick single-crystalline Ce:YIG layer epitaxially grown on a $<111>$-oriented nonmagnetic garnet (SGGG) substrate. (b) X-ray diffraction spectrum of the Ce:YIG/SGGG wafer and (c) its linear absorption spectrum.

absorption spectrum of the sample is shown in Fig. 1(c). At the fundamental wavelength of $800 \mathrm{~nm}$, there is no significant absorption, whereas it sharply increases around 400 $\mathrm{nm}$, suggesting that incident femtosecond pulses centered at $800 \mathrm{~nm}$ is absorbed by the multi-photon process. In the successive magneto-optical polar Kerr effect measurement, we also confirmed that the as-grown sample exhibited a weak coercive force of $30 \mathrm{Oe}$.

For all experimental results reported here, a modelocked Ti:Sappphire femtosecond laser system (SpectraPhysics, Tsunami with Millennia Vs) was used as a light source. The center wavelength, pulse width, and repetition rate were $800 \mathrm{~nm}, 80 \mathrm{fs}$, and $82 \mathrm{MHz}$, respectively. The direct laser writing was carried out using an inverted microscope (Olympus, IX71) where the beam from the laser system was tightly focused into the Ce:YIG layer using an objective lens $(40 \times, \mathrm{NA}=0.9)$. The focused laser beam was then scanned two-dimensionally (x-y scanning) using a closed-loop-controlled two-axis stage (Sigma Koki, BIOS$402 \mathrm{~T}$ with FC-501G) with a lateral resolution of $10 \mathrm{~nm}$. The longitudinal (z) position of the laser spot was also controlled by translating the objective lens using a computercontrolled motor stage (Chuo Precision Industrial, MSSFU) with a resolution of $62.5 \mathrm{~nm}$. The fabrication process was continuously monitored from the backside of the wafer (SGGG side) using a CCD camera (SONY, XC-77).

As shown in Figure 2(a), Ce:YIG was exposed by scanning the laser spot with a constant speed of 100 $\mu \mathrm{m} / \mathrm{min}$ for different irradiation powers ranged from 30 to $77 \mathrm{~mW}$. Figs. 2(b) and 2(c) are the reflected and phasecontrast optical microscope images of the fabricated line patterns, which are five single-pass lines with different powers [(i) - (v)], and a multi-pass line (20 scans) with 48 $\mathrm{mW}[(\mathrm{vi})]$. When the laser power was $60 \mathrm{~mW}$ or higher, thermal ablations in the Ce:YIG layer were observed, shown by the two black lines in Fig. 2(b) and by the two (a)
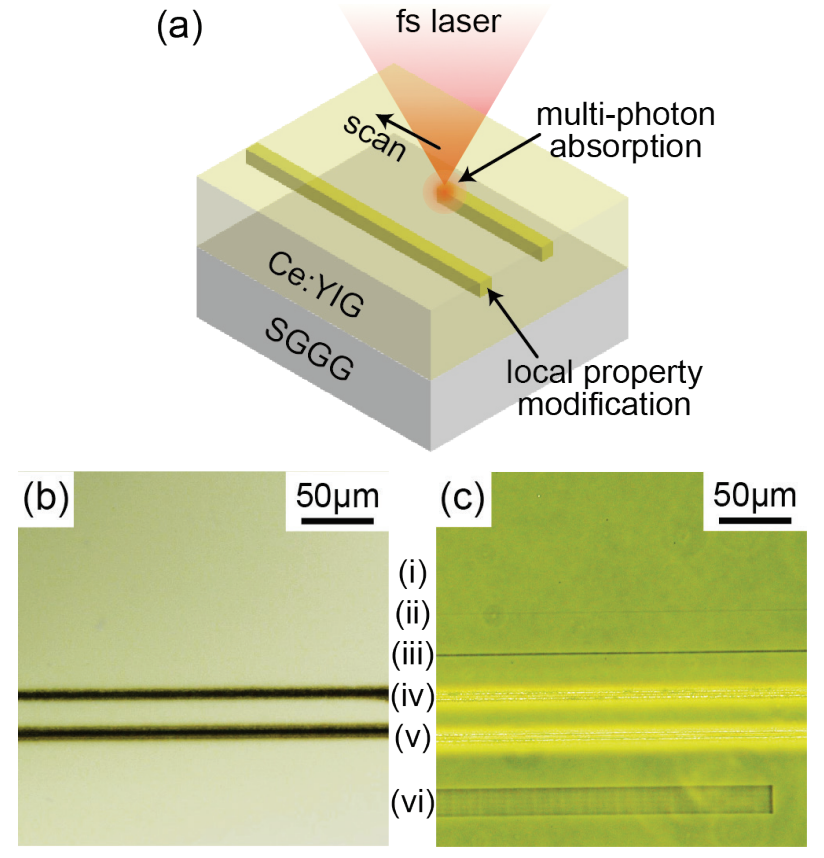

Fig. 2 (a) Schematic of the direct laser writing in a Ce:YIG layer on a SGGG wafer with a scanning speed of $100 \mu \mathrm{m} / \mathrm{min}$. (b) Reflected and (c) phase-contrast optical microscope images of the sample irradiated by single scanning with a laser power of (i) 30 $\mathrm{mW}$, (ii) $38 \mathrm{~mW}$, (iii) $48 \mathrm{~mW}$, (iv) $60 \mathrm{~mW}$, and (v) $77 \mathrm{~mW}$, and (vi) by multiple (20) scanning with $48 \mathrm{~mW}$.

yellow lines in Fig. 2(c). At moderate powers, the color phase of Ce:YIG was just changed from yellowish to darkish green without any cracking [two thin lines (ii) and (iii) in Fig. 2(c)], suggesting its optical properties were modified by the laser irradiation. The measured width of these two lines, (ii) and (iii), were $750 \mathrm{~nm}$ and $1.5 \mu \mathrm{m}$, which can be applicable to optical waveguides at infrared wavelengths On the other hand, for the laser power of $30 \mathrm{~mW}$ or lower, no remarkable change was visible in the optical microscope images.

\section{Optical and magnetic characterizations}

Our goal in this study is to show that, unlike conventional materials used in femtosecond laser processing, such as glasses and polymers, it is possible to control both the optical and magnetic properties of the exposed Ce:YIG layer. In the following, by quantitatively evaluating the refractive index and magnetization of the exposed Ce:YIG layer, we investigated the effect of laser irradiation on the Ce:YIG properties.

\subsection{Quantitative phase imaging}

The change in refractive index of the exposed Ce:YIG layer is most important property for optical waveguide applications. To this end, we firstly measured the refractive index change by employing a quantitative phase imaging (QPI) technique [28]. The QPI is a state-of-the-art technology, based on optical interferometry, to map the image of path-length shifts associated with the specimen. This image reveals quantitative information about both the local thickness and refractive index of the structure. By comparing QPI information between the exposed Ce:YIG with that for an as-grown Ce:YIG reference, the intracrystal refractive 


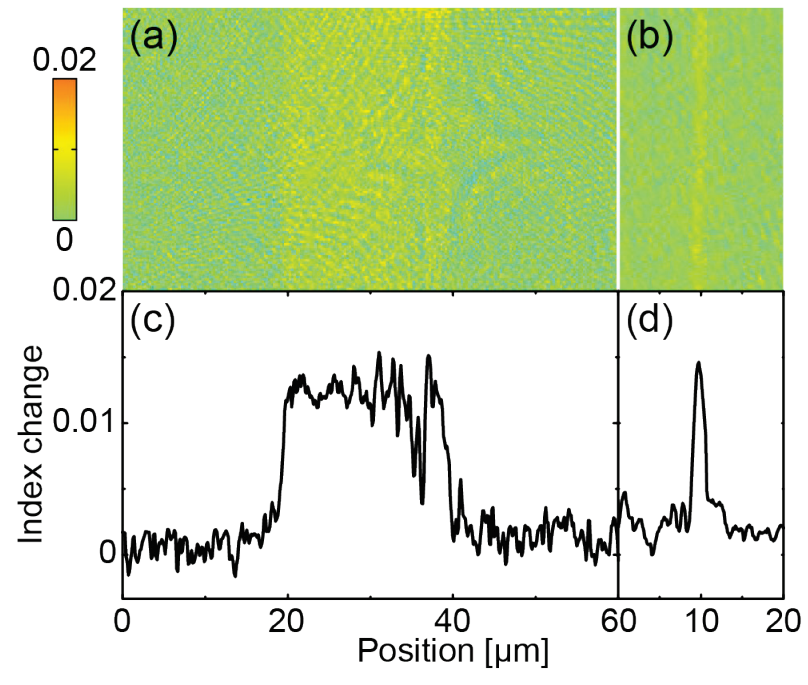

Fig. 3 In-plane distribution of refractive index change of the exposed Ce:YIG lines measured with a quantitative phase imaging technique. (a) and (b) correspond to (vi) and (ii) in Fig. 2(c), respectively. (c) and (d) are the averaged profiles of the refractive index change in the measured regions of (a) and (b).

index of the exposed Ce:YIG can be determined [29,30]. The refractive index and the thickness of the non-exposed Ce:YIG was measured beforehand with an ellipsometer and scanning electron microscope. Figures 3(a) and 3(b) are the QPI images measured at a wavelength of $632.8 \mathrm{~nm}$, showing the in-plane distribution of the refractive index change of the fabricated line patterns [(vi) and (ii) in Fig. 2(c)]. From the images, the refractive index of the exposed regions was quantitatively determined and increased by $0.015 \pm 0.001(0.7 \%$ of Ce:YIG refractive index). To form an optical waveguide in the Ce:YIG with the refractive index change of $0.7 \%$, a waveguide width has to be $10 \mu \mathrm{m}$ or more for sufficient optical confinement. On the other hand, the edge-transition width of the fabricated line is less than $500 \mathrm{~nm}$, which is much smaller than the waveguide width, ensuring no unwanted effect on the wave-guiding performance.

\subsection{Magneto-optical polar Kerr effect measurement}

The change in magnetization of the exposed Ce:YIG layer is another important property for novel magneto-optical device applications. To characterize the magnetic-domain configuration in the Ce:YIG layer, the magneto-optical polar Kerr effect (MOKE) measurement was carried out. Figure 4 shows the schematic magnetization $(\mathrm{M}-\mathrm{H})$ curve of the Ce:YIG layer and the corresponding MOKE images measured with different external magnetic field. In Fig. 4(a), the blue curve is for the exposed region, and the red curve is for the non-exposed one, and points (i) - (vi) on the curves correspond to MOKE images (i) - (vi), respectively. Note that this $\mathrm{M}-\mathrm{H}$ curve was estimated from the magneticdomain configurations of each MOKE image and is given simply to assist the understanding of the magnetic properties of MOKE images. The MOKE images were measured using a mercury lamp at room temperature with an external magnetic field (parallel to the surface layer). The dark regions are positively magnetized, and the light regions are negatively magnetized.

Firstly, the sample was measured by increasing the magnetic field from -500 Oe to 500 Oe. At an external magnetic field of $-470.7 \mathrm{Oe}$, the entire Ce:YIG layer was negatively magnetized [(i) in Fig. 4]. This means that the Ce:YIG layer, including irradiated regions, was magnetized in the same direction as the magnetic field. As magnetic field increased in the positive direction, the MOKE images changed from (ii) to (iii). The exposed regions easily changed their magnetization in response to the external magnetic field. For the non-exposed regions, on the other hand, the magnetization was maintained. Note that no phase change were observed for the upper two lines because their magnetic property was erased due to the ablation. At an external magnetic field of 495 Oe, the entire Ce:YIG layer was positively magnetized [(iv) in Fig. 4]. Then, the sample was measured by sweeping the magnetic field from the positive side (500 Oe) to the negative side $(-500 \mathrm{Oe})$ and observed that the magnetic-domain showed a hysteretic cycling [(v) and (vi) in Fig. 4]. Form these results, we concluded that the laser irradiation induced dramatic change in the magnetic property of the Ce:YIG layer from hard to soft, thereby locally controlling the magnetization inside the exposed Ce:YIG layer.

\section{Conclusions and outlook}

We have demonstrated the femtosecond laser fabrication of optical and magnetic microstructures in Ce:YIG. The laser irradi-
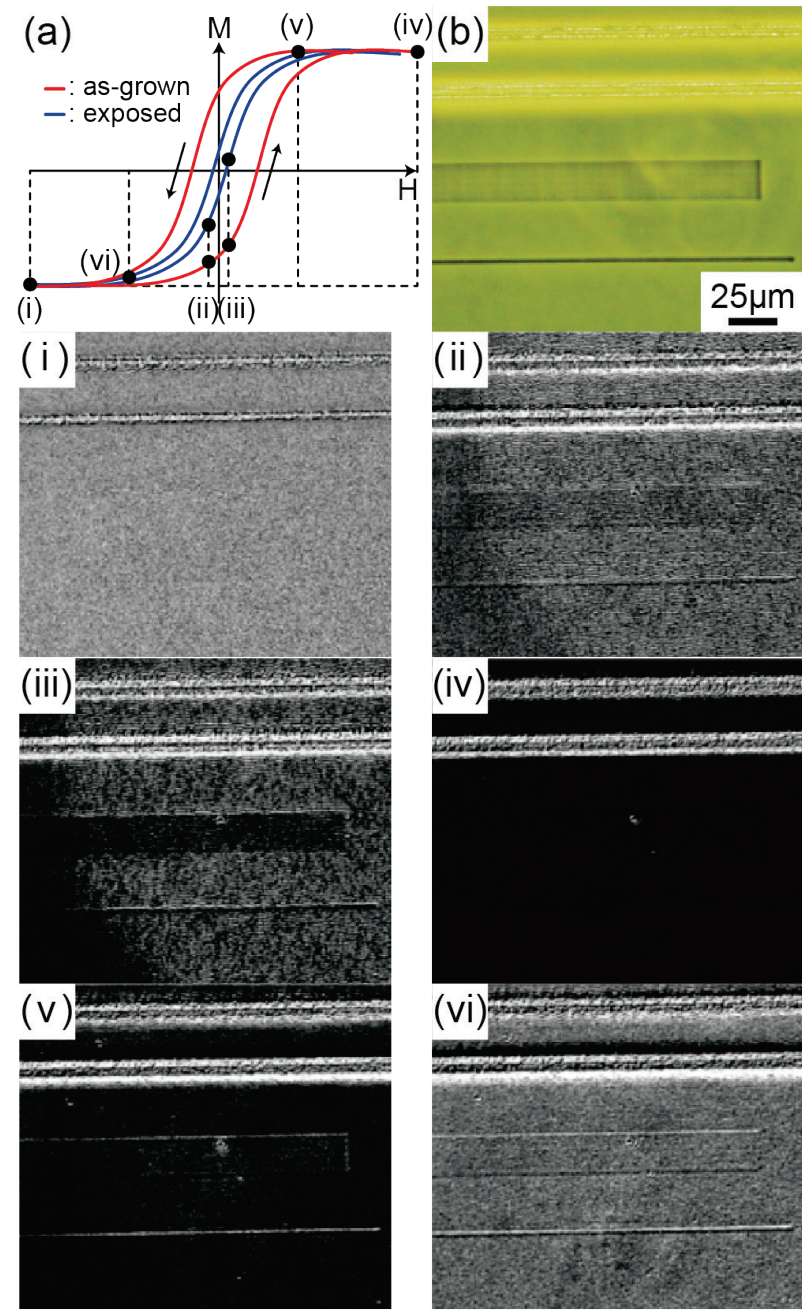

Fig. 4 (a) Schematic of magnetization (M-H) curves of a Ce:YIG layer with and without the irradiation. (b) Phase-contrast microscope image of the measured area and the corresponding magneto-optical polar-Kerr-effect images measured with an external magnetic field of (i) $-470.7 \mathrm{Oe}$, (ii) $-25.3 \mathrm{Oe}$, (iii) $24.3 \mathrm{Oe}$, (iv) $495 \mathrm{Oe},(\mathrm{v}) 197.9 \mathrm{Oe}$, and (vi) $-223.5 \mathrm{Oe}$. 

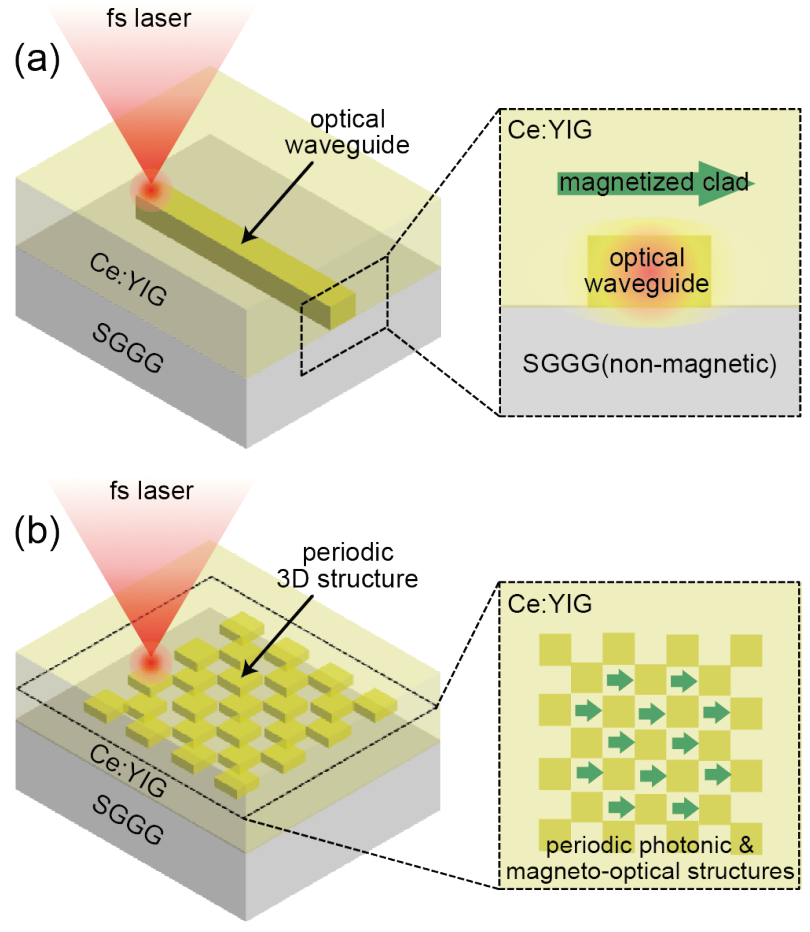

Fig. 5 Functional 3D micro/nanostructures monolithically fabricated in a Ce:YIG layer on a SGGG substrate: (a) nonreciprocalphase-shift waveguide and (b) 3D magneto-photonic crystal.

ation induced local changes in both optical and magnetic properties of Ce:YIG. The QPI technique proved that the refractive index was increased by $0.7 \%$, and the MOKE measurement showed that the magnetization property is changed from hard to soft, decreasing the coercivity. Possible mechanism for the change of properties could be a state change of $\mathrm{Ce}$ ions or a thermal annealing effect, e.g., crysalline-to-amorphous transformation, but detailed experiments are needed to clarify the cause. These results demonstrates the usefulness of the direct laser writing in Ce:YIG and other magneto-optical materials; Magneto-optical elements combined with semiconductor optical components, e.g., optical waveguides, can be incorporated monolithically into one device.

For future outlook, two potential applications of the direct laser writing in Ce:YIG are discussed in Figure 5. A nonreciprocalphase-shift waveguide [Fig. 5 (a)] can be fabricated by simply scanning a laser spot in magneto-optical materials [31]. The upper cladding layer on the waveguide is left non-exposed to exhibit a large residual magnetization. The resultant device works as a nonreciprocal-phase-shift waveguide for an integrated optical isolator in the form of a Mach-Zehnder interferometer. A magneto-photonic crystal (MPC) [Fig. 5 (b)] can be also constructed by simply writing $3 \mathrm{D}$ micro/nanostructures to form a spatially periodic refractive-index change inside the Ce:YIG [27]. The magnetization can be controlled space-periodically with an appropriate external magnetic field. As a result, a periodic optical and magnetic structures can be realized in the Ce:YIG layer. With this magneto-photonic crystal, more flexible band engineering can be achieved. Our new fabrication technique presented here will open new fields in the technology of magneto-optical devices and contribute to the development of novel 3D magneto-optical devices for optical communication and photonic applications.

\section{Acknowledgments}

The authors acknowledge financial support from the Ministry of Education, Culture, Sports, Science and Technology (MEXT); JSPS KAKENHI Grant Nos. 24246061, 24656046, 25420321, 22109006, 23710160, 24760270, 23000010, 24686040, and the Strategic Information and Communications R\&D Promotion Programme (SCOPE) of the Ministry of Internal Affairs and Communications.

\section{References}

[1] P.-W. Wu, W. Cheng, I. B. Martini, B. Dunn, B. J. Schwartz, and E. Yablonovitch, Adv. Mater., 12, (2000) 1438.

[2] W. Zhou, S. M. Kuebler, K. L. Braun, T. Yu, J. K. Cammack, C. K. Ober, J. W. Perry, and S. R. Marder, Science, 296, (2002) 1106.

[3] T. Tanaka, A. Ishikawa, and S. Kawata: Appl. Phys. Lett., 88, (2006) 081107.

[4] A. Ishikawa, T. Tanaka, and S. Kawata: Appl. Phys. Lett., 89, (2006) 113102.

[5] M. Goppert-Mayer, Ann. Phys., 9, (1931) 273.

[6] S. Kawata, H.-B. Sun, T. Tanaka, and K. Takada, Nature, 412, (2001) 697.

[7] Y.-Y. Cao, N. Takeyasu, T. Tanaka, X.-M. Duan, and S. Kawata: Small 5, (2009) 1144.

[8] K. M. Davis, K. Miura, N. Sugimoto, and K. Hirao: Opt. Lett., 21, (1996) 1729.

[9] S. Nolte, M. Will, J. Burghoff, and A. Tuennermann: Appl. Phys. A, 77, (2003) 109.

[10] S. L. Chin, S. A. Hosseini, W. Liu, Q. Luo, F. Théberge, N. Aközbek, A. Becker, V. P. Kandidov, O. G. Kosareva, and H. Schroeder: Can. J. Phys., 83, (2005) 863.

[11]C. B. Schaffer, A. Brodeur, J. F. Garcia, and E. Mazur: Opt. Lett. 26, (2011) 93.

[12] A. Ishikawa and T. Tanaka: J. Laser Micro/Nanoeng., $7,(2012) 11$

[13] A. Ishikawa, T. Tanaka, and S. Kawata: Appl. Phys. Lett. 91, (2007) 113118.

[14] M. S. Rill, C. Plet, M. Thiel, I. Staude, G. V. Freymann, S. Linden, and M. Wegener: Nat. Mater., 7, (2008) 543.

[15] A J. K. Gansel, M. Thiel, M. S. Rill, M. Decker, K. Bade, V. Saile, G. V. Freymann, S. Linden, and M. Wegener, Science, 325, (2009) 1513.

[16] A. Ishikawa and T. Tanaka: IEEE J. Select. Topics Quantum Electron., 19, (2013) 4700110.

[17] T. Amemiya, A. Ishikawa, Y. Shoji, P. N. Hai, M. Tanaka, T. Mizumoto, T. Tanaka, and S. Arai: Opt. Lett., 39, (2014) 212.

[18] T. Boudiar, B. Payet-Gervy, M.-F. Blanc-Mignon, J.-J. Rousseau, M. Le Berre, and H. Joisten: J. Magn. Magn. Mater., 284, (2004) 77.

[19] M. C. Sekhar, J. Y. Hwang, M. Ferrera, Y. Linzon, L. Razzari, C. Harnagea, M. Zaezjev, A. Pignolet, and R. Morandotti: Appl. Phys. Lett., 94, (2009) 181916.

[20] A. K. Zvezdin and V. A. Kotov: "Modern Magnetooptics and Magnetooptical Materials" (Taylor and Francis, 1997).

[21] H. Dötsch, N. Bahlmann, O. Zhuromskyy, M. Hammer, L. Wilkens, R. Gerhardt, P. Hertel, and A. F. Popkov: J. Opt. Soc. Am. B, 22, (2005) 240. 
[22] T. Mizumoto, R. Takei, and Y. Shoji: IEEE J. Quantum Electron., 48, (2012) 252.

[23]Y. Shoji, T. Mizumoto, H. Yokoi, I.-W. Hsieh, and R. M. Osgood Jr.: Appl. Phys. Lett., 92, (2008) 071117.

[24] M.-C. Tien, T. Mizumoto, P. Pintus, H. Kromer, and J. E. Bowers: Opt. Express 19, (2011) 11740.

[25] S. Ghosh, S. Keyvaninia, Y. Shirato, T. Mizumoto, G. Roelkens, and R. Baets: IEEE Photon. J., 5, (2013) 6601108.

[26]R. Takei, K. Yoshida, and T. Mizumoto: Jpn. J. Appl. Phys., 49, (2010) 086204.

[27]I. L. Lyubchanskii, N. N. Dadoenkova, M. I. Lyubchanskii, E. A. Shapovalov, and Th. Rasing: J. Phys. D, 36, (2003) R277.

[28] http://www.piphotonics.co.jp/EN/qpm/index.html

[29] H. Iwai, C. Fang-Yen, G. Popescu, A. Wax, K. Badizadegan, R. R. Dasari, and M. S. Feld: Opt. Lett., 29, (2004) 2399.

[30] T. Yamauchi, H. Iwai, M. Miwa, and Y. Yamashita: Opt. Express, 16, (2008) 12227.

[31]H. Yokoi, T. Mizumoto, N. Shinjo, N. Futakuchi, and Y. Nakano: Appl. Optics, 39, (2000) 6158.

(Received: June 16, 2014, Accepted: December 15, 2014) 